\title{
Long non-coding RNA PVT1 is associated with tumor progression and predicts recurrence in hepatocellular carcinoma patients
}

\author{
CHAOFENG DING ${ }^{1,2^{*}}$, ZHE YANG $^{1 *}$, ZHEN LV $^{2 *}$, CHENGLI DU ${ }^{2}$, HENG XIAO $^{2}$, CHUANHUI PENG $^{1}$, \\ SHAOBING CHENG ${ }^{2}$, HAIYANG XIE ${ }^{1,2}$, LIN ZHOU $^{1,2}$, JIAN WU $^{1,2}$ and SHUSEN ZHENG ${ }^{1,2}$ \\ ${ }^{1}$ Division of Hepatobiliary and Pancreatic Surgery, Department of Surgery, The First Affiliated Hospital, School of Medicine, \\ Zhejiang University; ${ }^{2}$ Key Laboratory of Combined Multi-Organ Transplantation, \\ Ministry of Public Health, Hangzhou, Zhejiang 310003, P.R. China
}

Received February 8, 2014; Accepted October 23, 2014

DOI: $10.3892 / 01.2014 .2730$

\begin{abstract}
PVT1, which maps to chromosome 8q24, is a copy number amplification-associated long non-coding RNA. Overexpression of PVT1 is a powerful predictor of tumor progression and patient survival in a diverse range of cancer types. However, the association between PVT1 and hepatocellular carcinoma (HCC) remains unclear. The aim of the present study was to examine the expression pattern of PVT1, and its clinical significance in HCC. Between 2003 and 2012, reverse transcription-quantitative polymerase chain reaction was used to determine the expression levels of PVT1 in two independent cohorts: Cohort one, 58 HCC resection samples; and cohort 2, 214 HCC transplant samples. Additionally, the correlation between PVT1 expression levels and clinical parameters and outcomes was analyzed. The relative expression levels of PVT1 were significantly higher in cancerous tissues compared with the corresponding non-cancerous tissues (cohort one, $\mathrm{P}=0.0016$; cohort two, $\mathrm{P}=0.0274$ ). Furthermore, overexpression of PVT1 was associated with a higher serum $\alpha$-fetoprotein expression level $(\mathrm{P}=0.011)$ and a higher recurrence rate $(\mathrm{P}=0.004)$. Kaplan-Meier analysis indicated that the patients with high PVT1 expression exhibited poor recurrence-free survival $(\mathrm{P}=0.021)$, and multivariate analysis demonstrated that high levels of PVT1 expression are an independent predictor for HCC recurrence $(\mathrm{P}=0.042$; hazard ratio, 1.653). Thus, the high expression levels of PVT1
\end{abstract}

Correspondence to: Professor Shusen Zheng or Professor Jian Wu, Division of Hepatobiliary and Pancreatic Surgery, Department of Surgery, The First Affiliated Hospital, School of Medicine, Zhejiang University, 79 Qingchun Road, Hangzhou, Zhejiang 310003, P.R. China

E-mail: shusenzheng@zju.edu.cn

E-mail: drwujian@hotmail.com

*Contributed equally

Key words: hepatocellular carcinoma, long non-coding RNA, PVT1, recurrence, progression in HCC may serve as a novel biomarker for predicting tumor recurrence in HCC patients, and as a potential therapeutic target.

\section{Introduction}

Hepatocellular carcinoma (HCC) represents the fifth most prevalent malignancy and the second most common cause of cancer-related mortality worldwide, with $\sim 695,900$ mortalities per year (1). Half of these cases occur in China, due to high incidence of chronic hepatitis B virus (HBV) infection (2). Liver surgery, including liver resection and liver transplantation, are considered to be curative treatment strategies, as they provide complete oncologic resection (R0 resection). However, tumor recurrence following liver surgery remains a critical issue (recurrence rate, $30-50 \%$ ), compromising the long-term survival of patients (3-5). The molecular mechanisms underlying HCC progression remain poorly understood. Therefore, investigating ideal biomarkers for the improved prediction of postoperative recurrence may aid surgeons in selecting patients or adopting preventive strategies for patients at high risk of recurrence.

Previously, RNA sequencing revealed a novel class of transcripts termed long non-coding RNAs (lncRNAs). LncRNAs are $>200$ nucleotides long and lack protein-coding potential, thus, they were previously regarded as random transcriptional noises. However, increasing evidence has implicated lncRNAs in critical regulatory roles in cancer biology (6-8). LncRNAs have been demonstrated to control gene expression through transcriptional and posttranscriptional regulation (9). Various classic lncRNAs have been characterized in human hepatocarcinogenesis as having oncogenic and tumor suppressive roles, such as HOX transcript antisense RNA (HOTAIR) $(10,11)$, metastasis-associated lung adenocarcinoma transcript 1 (MALAT1) (12), maternally expressed 3 (MEG3) (13) and H19 (14). Our previous studies have demonstrated that overexpression of HOTAIR (10) and MALAT1 (12) exhibit oncogenic properties, and may serve as independent prognostic factors for HCC. Furthermore, MEG3 was frequently downregulated in HCC and inhibited cell growth by functionally interacting with p53 (13). Additionally, H19 suppressed HCC metastasis by epigenetic activation of the microRNA (miR)200 family (14). 
These data support the hypothesis that MG3 and H19 act as tumor suppressors in HCC.

PVT1, which maps to chromosome 8q24, is a copy number amplification-associated lncRNA. Overexpression of PVT1 is a powerful predictor of tumor progression and patient survival in colorectal (15), ovarian and breast cancers (16). Furthermore, PVT1 exerts regulatory functions in various biological processes, such as proliferation, apoptosis, mobility and invasion $(15,16)$, and chromosome $8 \mathrm{q} 24$ is a commonly amplified region in $\mathrm{HCC}(17,18)$. However, the association between PVT1 and HCC remains unclear.

The aim of the present study was to examine the expression pattern of PVT1 and its clinical significance in HCC.

\section{Materials and methods}

Patients and surgical specimens. Fifty-eight snap-frozen HCC tissues and the corresponding non-cancerous tissues were obtained from patients undergoing liver resection at the First Affiliated Hospital of Zhejiang University (Hangzhou, China) between 2009 and 2012 (cohort one). An additional 214 HCC tissues were collected from patients undergoing liver transplantation at the First Affiliated Hospital of Zhejiang University between 2003 and 2012 (cohort two), and were used for survival analysis and validation. The liver tissue specimens were immediately frozen in liquid nitrogen following surgical resection and stored at $-80^{\circ} \mathrm{C}$ prior to the extraction of total RNA. A postoperative histopathological examination by experienced pathologists was used to establish a diagnosis of HCC in these patients. The histological grade of differentiation was evaluated on hematoxylin and eosin-stained sections according to the Edmondson-Steiner grading method (19). Complete clinical and laboratory data were collected in a perspective database. The tumor staging was defined according to the sixth edition of the tumor-node-metastasis (TNM) classification system published by the American Joint Committee on Cancer and the Union for International Cancer Control (20). The requirements for selecting transplant candidates varies depending on the criteria, for example: The Milan criteria defines HCC as a single tumor $\leq 5 \mathrm{~cm}$ or up to three tumors $\leq 3 \mathrm{~cm}$ (21); the University of California, San Francisco (UCSF) criteria defines HCC as a single nodule $\leq 6.5 \mathrm{~cm}$ or up to three nodules $\leq 4.5 \mathrm{~cm}$, and total tumor diameter $\leq 8 \mathrm{~cm}(22)$; and the Hangzhou criteria defines HCC as a total tumor diameter of $\leq 8 \mathrm{~cm}$, or a total tumor diameter of $>8 \mathrm{~cm}$, with a well or moderately differentiated histopathological grade and a preoperative serum AFP level of $\leq 400 \mathrm{ng} / \mathrm{ml}$, simultaneously (23).

The present study was conducted with the approval of the Institutional Review Board and Ethics Committee of the First Affiliated Hospital, Zhejiang University (Hangzhou, China) and in accordance with the Declaration of Helsinki. Thus, the study conformed to international and national regulations. Informed consent was obtained from all of the patients.

Follow-up. Patient follow-up was conducted every 2-3 months during the first two years following surgery and 3-6 months thereafter. The endpoint of study was September 3, 2013. During the follow-up period, all patients were monitored using abdomen ultrasonography, chest X-ray, emission computed tomography and serum AFP tests. Following a suspected recurrence, computed tomography, magnetic resonance imaging or positron emission tomography-computed tomography, were immediately performed. These imaging techniques were required for a new lesion to be detected and recurrence to be diagnosed; an increase in serum AFP levels alone was not regarded as recurrence. Once recurrent tumors were diagnosed, treatment was implemented based on the tumor size, number, location and vascular invasion, as well as the liver function. The recurrence-free survival (RFS) period was calculated from the date of surgery to the date of detection of tumor recurrence, mortality or the most recent observation. All follow-up examinations were performed by two physicians who were unaware of the study. The mean follow-up time was 27.58 months (range, 2-124 months).

Cell culture. The healthy liver cell line HL7702, the liver cancer cell lines Huh7, SK-hep1, SMMC-7721, HepG2, Hep3B, PLC/PRF/5 and Bel-7402, as well as the metastasis-capable human HCC cell lines MHCC97L, MHCC97H and HCCLM3, were purchased from the American Type Culture Collection (Manassas, VA, USA), the Shanghai Institute of Biochemistry and Cell Biology (Shanghai, China), and the Liver Cancer Institute of Fudan University (Shanghai, China), respectively. All of the cell lines were maintained in Dulbecco's modified Eagle's medium with high glucose or RPMI-1640, containing $10 \%$ fetal bovine serum, and cultured in a humidified $5 \% \mathrm{CO}_{2}$ incubator at $37^{\circ} \mathrm{C}$.

RNA extraction and quantitative reverse transcription-quantitative polymerase chain reaction (RT-qPCR). Total RNAs from the specimens and cell lines were extracted using TRIzol reagent (Invitrogen Life Technologies, Carlsbad, CA, USA); and complementary DNA was synthesized using Moloney murine leukemia virus reverse transcriptase (Promega Corporation, Madison, WI, USA) according to the manufacturer's instructions. The expression of PVT1 was determined using qPCR, which was performed on an Applied Biosystems 7500 Fast Real-Time PCR system (Applied Biosystems Life Technologies, Foster City, CA, USA) and SYBR ${ }^{\circledR}$ Green dye (Takara Biotechnology Co., Inc., Dalian, China). All PCRs were performed in triplicate and GAPDH was used to normalize mRNA expression levels. Relative quantification was performed using the comparative threshold cycles $\left(2^{-\Delta \Delta C t}\right.$ method) as described in the manufacturer's instructions. The primer sequences were as follows: Sense, 3'-CATCCGGCGCTCAGCT-5' and antisense, 3'-TCATGATGGCTGTATGTGCCA-5' for PVT1; and sense, 3'-ATGGGGAAGGTGAAGGTCG-5' and antisense, 3'-GGGGTCATTGATGGCAACAATA-5' for GAPDH.

Statistical analysis. Comparisons of continuous data were analyzed using the independent t-test between the two groups, whereas categorical data were analyzed by the $\chi^{2}$ test. Comparisons of continuous data among multiple groups were calculated using one-way analysis of variance. A receiver operating characteristic (ROC) curve was used to determine the cut-off value of PVT1 expression that yielded the highest combined sensitivity and specificity for discriminating between patients exhibiting HCC recurrence and those not 
Table I. Clinical characteristics of HCC patients.

\begin{tabular}{|c|c|c|c|}
\hline & Cohort one $(n=58)$ & Cohort two $(n=214)$ & P-value \\
\hline Age, years & & & $0.000^{\mathrm{a}}$ \\
\hline Median & 60 & 49 & \\
\hline Range & $26-86$ & $20-71$ & \\
\hline Gender, n (\%) & & & 0.951 \\
\hline Female & $5(8.62)$ & $19(8.88)$ & \\
\hline Male & $53(91.38)$ & $195(91.12)$ & \\
\hline $\mathrm{HBV}, \mathrm{n}(\%)$ & & & $0.000^{\mathrm{a}}$ \\
\hline Negative & $11(18.97)$ & $6(2.34)$ & \\
\hline Positive & $47(81.03)$ & 209 (97.66) & \\
\hline Cirrhosis, n (\%) & & & $0.000^{\mathrm{a}}$ \\
\hline No & $22(37.93)$ & $2(0.93)$ & \\
\hline Yes & $36(62.07)$ & $212(99.07)$ & \\
\hline Tumor size, n (\%) & & & 0.059 \\
\hline$\leq 5 \mathrm{~cm}$ & $25(43.10)$ & $122(57.01)$ & \\
\hline$>5 \mathrm{~cm}$ & $33(56.90)$ & $92(42.99)$ & \\
\hline Tumor number, n (\%) & & & $0.000^{\mathrm{a}}$ \\
\hline$=1$ & $52(89.66)$ & $89(41.59)$ & \\
\hline$>1$ & $6(10.34)$ & $125(58.41)$ & \\
\hline PVTT, n (\%) & & & $0.000^{\mathrm{a}}$ \\
\hline Negative & $54(93.10)$ & $142(66.36)$ & \\
\hline Positive & $4(6.90)$ & $72(33.64)$ & \\
\hline AFP, n (\%) & & & 0.270 \\
\hline$\leq 400 \mathrm{ng} / \mathrm{ml}$ & $34(58.62)$ & $108(50.47)$ & \\
\hline$>400 \mathrm{ng} / \mathrm{ml}$ & $24(41.38)$ & $106(49.53)$ & \\
\hline Histopathological grade, $\mathrm{n}(\%)$ & & & 0.512 \\
\hline Well + moderately & $27(46.55)$ & $110(51.40)$ & \\
\hline Poorly & $31(53.45)$ & $104(48.60)$ & \\
\hline TNM stage, n (\%) & & & $0.000^{\mathrm{a}}$ \\
\hline I + II & $52(89.66)$ & $99(46.26)$ & \\
\hline III + IV & $6(10.34)$ & $115(53.74)$ & \\
\hline Milan criteria (19), n (\%) & & & 0.374 \\
\hline Within criteria & $24(41.38)$ & $75(35.05)$ & \\
\hline Beyond criteria & $34(58.62)$ & $139(64.95)$ & \\
\hline UCSF criteria (20), n (\%) & & & 0.175 \\
\hline Within criteria & $31(53.45)$ & $93(43.46)$ & \\
\hline Beyond criteria & $27(46.55)$ & $121(56.54)$ & \\
\hline Hangzhou criteria (21), n (\%) & & & $0.000^{\mathrm{a}}$ \\
\hline Within criteria & $46(79.31)$ & $105(49.07)$ & \\
\hline Beyond criteria & $12(20.69)$ & $109(50.93)$ & \\
\hline
\end{tabular}

${ }^{\mathrm{a}} \mathrm{P}<0.05$ was considered to indicate a statistically significant difference, using the independent samples t-test for age and the $\chi^{2}$ test for all other data. HCC, hepatocellular carcinoma; HBV, hepatitis B virus; PVTT, portal vein tumor thrombosis; AFP, serum $\alpha$-fetoprotein; TNM, tumor-node-metastasis; UCSF, University of California, San Francisco.

exhibiting recurrence. Recurrence-free survival was analyzed using the Kaplan-Meier method and compared by performing the log-rank test. Independent prognostic factors were assessed in the univariate and multivariate analysis using the Cox proportional-hazards regression model. All statistical analyses were performed using SPSS for Windows (version 16.0; SPSS, Inc., Chicago, IL, USA) and GraphPad Prism (version 5.0; GraphPad
Software, Inc., La Jolla, CA, USA) software. P<0.05 was considered to indicate a statistically significant difference.

\section{Results}

Characteristics of HCC patients. Snap-frozen HCC tissues were obtained from $272 \mathrm{HCC}$ patients who had undergone 
A

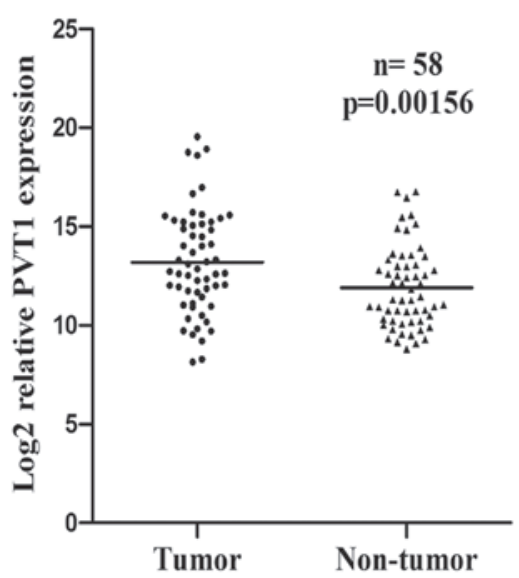

B

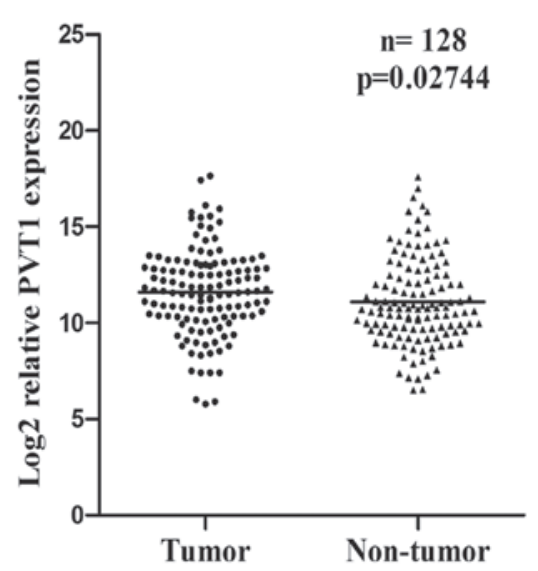

Figure 1. PVT1 expression levels in hepatocellular carcinoma tumor (HCC) tissue and the corresponding non-tumorous tissue. PVT1 expression levels were normalized to an internal control and $\log 2$-transformed. (A) Cohort one consists of 58 paired HCC resection samples and (B) cohort two consists of 128 paired HCC transplant samples. Horizontal lines in the plots represent the mean values. P-values between samples were obtained by performing a paired t-test.

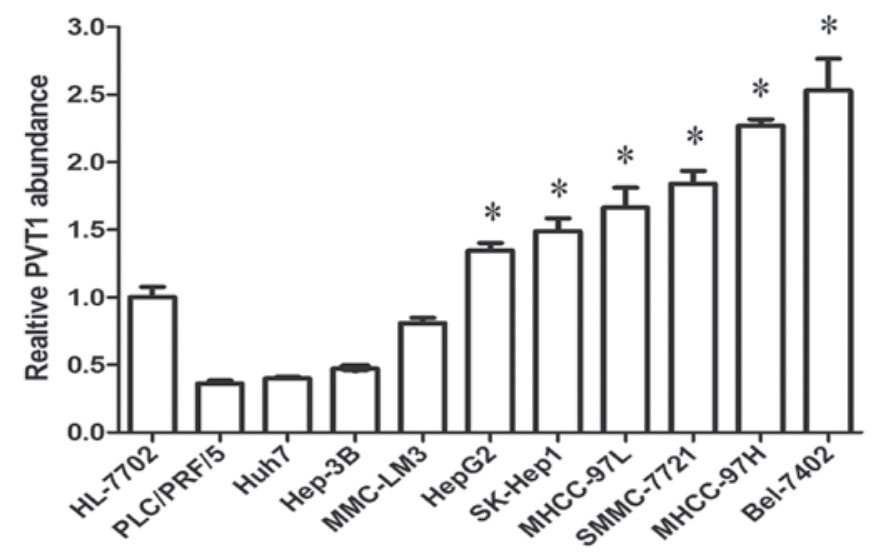

Figure 2. Relative PVT1 expression levels in 10 liver cancer cell lines. HL-7702 is a healthy liver cell line. Data are the mean \pm standard error of the mean $(n=3)$. ${ }^{*} \mathrm{P}<0.05$, vs. the control (HL-7702 cells), according to the paired t-test.

liver surgery at the First Affiliated Hospital of Zhejiang University between 2003 and 2012. The present study involved two independent cohorts of HCC patients: Cohort one included $58 \mathrm{HCC}$ patients who had undergone radical resection between 2009 and 2012; while cohort two included 214 HCC patients who had received a liver transplant between 2003 and 2012.

The majority of the HCC patients in the two cohorts were male $(91.18 \%)$ with a tumor size of $>5 \mathrm{~cm}$ at the time of surgery (45.96\%), an elevated serum AFP level (47.79\%) and with tumors exceeding the Milan and UCSF criteria (63.60 and 54.41\%, respectively; Table I). In addition, the patients in cohort two were HCC transplant patients, thus representing a group of patients with advanced disease. Furthermore, the patients in cohort two were relatively younger than those in cohort one, and more patients in cohort two exhibited HBV infection, liver cirrhosis, multifocal tumor, portal vein tumor thrombosis (PVTT) and advanced TNM stage.

PVTl overexpression in the two independent HCC cohorts and liver cancer cell lines. In the present study, the expression levels of PVT1 in the 272 HCC patients from the two independent cohorts were determined using RT-qPCR. Compared with the corresponding non-tumor liver tissues of the HCC cohorts, PVT1 expression was significantly increased in the cancerous tissues of the patients in cohort one $(\mathrm{P}=0.0016$; Fig. 1A) and cohort two ( $\mathrm{P}=0.0274$; Fig. 1B). Furthermore, the expression of PVT1 was defined in 10 liver cancer cell lines and one healthy liver cell line. Of the 10 liver cancer cell lines, six (HepG2, SK-Hep1, MHCC-97L, SMMC-7721, MHCC-97H and Bel-7402) expressed a higher level of PVT1 than the healthy liver cell line (Fig. 2). Thus, the present study determined that PVT1 expression was significantly increased in HCC.

Association between PVT1 and HCC progression. As PVT1 expression was significantly increased in $\mathrm{HCC}$, the association between PVT1 expression in HCC and disease progression was evaluated. First, the expression level of PVT1 in different TNM-stage patients was assessed, as TNM staging is a widely accepted system for HCC stratification. In HCC cohort two, advanced-stage patients (stages III and IV; $n=115$ ) exhibited increased expression levels of PVT1 compared with early-stage patients (stages I and II; n=99) ( $\mathrm{P}=0.0466$; Fig. 3A). Additionally, the patients exhibiting disease recurrence $(n=114)$ demonstrated higher levels of PVT1 expression compared with the non-recurrence recipients $(\mathrm{n}=100)(\mathrm{P}=0.0477$;Fig. 3B). However, in HCC cohort one, it was difficult to analyze the data with sufficient statistical power due to the limited number of patients with an advanced disease stage (Fig. 3C).

To investigate the clinicopathological correlation of PVT1 expression in HCC tissues, the patients were divided into high and low expression groups according to the cut-off value obtained from the ROC curve analysis. No significant correlations with any of the clinicopathological parameters tested were observed in HCC cohort one (Table II). In HCC cohort two, no significant correlations were identified between PVT1 expression and clinicopathological parameters such as age, gender, tumor number, tumor size, PVTT, histopathological grade or TNM stage (Table III). However, high PVT1 expression levels in HCC cohort two were significantly correlated with a higher AFP level $(\mathrm{P}=0.011)$ and 
Table II. Clinicopathological correlation of PVT1 expression in human HCC (cohort one).

\begin{tabular}{|c|c|c|c|c|c|c|}
\hline \multirow[b]{2}{*}{ Factors } & \multirow[b]{2}{*}{ Cases, $\mathrm{n}$} & \multicolumn{2}{|c|}{ Low PVT1 expression } & \multicolumn{2}{|c|}{ High PVT1 expression } & \multirow[b]{2}{*}{ P-value } \\
\hline & & $\mathrm{n}$ & $\%$ & $\mathrm{n}$ & $\%$ & \\
\hline \multicolumn{7}{|l|}{ Age, years } \\
\hline$\leq 60$ & 33 & 5 & 55.56 & 28 & 57.14 & 0.930 \\
\hline$>60$ & 25 & 4 & 44.44 & 21 & 42.86 & \\
\hline \multicolumn{7}{|l|}{ Gender } \\
\hline Female & 5 & 0 & 0.00 & 5 & 10.20 & 0.316 \\
\hline Male & 53 & 9 & 100.00 & 44 & 89.80 & \\
\hline \multicolumn{7}{|l|}{ HBV } \\
\hline Negative & 11 & 1 & 11.11 & 10 & 20.41 & 0.513 \\
\hline Positive & 47 & 8 & 88.89 & 39 & 79.59 & \\
\hline \multicolumn{7}{|l|}{ Cirrhosis } \\
\hline No & 22 & 4 & 44.44 & 18 & 36.73 & 0.661 \\
\hline Yes & 36 & 5 & 55.56 & 31 & 63.27 & \\
\hline \multicolumn{7}{|l|}{ Tumor size, $\mathrm{cm}$} \\
\hline$\leq 5$ & 25 & 2 & 22.22 & 23 & 46.94 & 0.169 \\
\hline$>5$ & 33 & 7 & 77.78 & 26 & 53.06 & \\
\hline \multicolumn{7}{|l|}{ Tumor number } \\
\hline Single & 52 & 8 & 88.89 & 44 & 89.80 & 0.935 \\
\hline Multiple & 6 & 1 & 11.11 & 5 & 10.20 & \\
\hline \multicolumn{7}{|l|}{ PVTT } \\
\hline Absent & 54 & 8 & 88.89 & 46 & 93.88 & 0.587 \\
\hline Present & 4 & 1 & 11.11 & 3 & 6.12 & \\
\hline \multicolumn{7}{|c|}{ Preoperative AFP level, ng/ml } \\
\hline$\leq 400$ & 34 & 7 & 77.78 & 27 & 55.10 & 0.204 \\
\hline$>400$ & 24 & 2 & 22.22 & 22 & 44.90 & \\
\hline \multicolumn{7}{|c|}{ Histopathological grade } \\
\hline Well + moderately & 27 & 5 & 55.56 & 22 & 44.90 & 0.556 \\
\hline Poorly & 27 & 4 & 44.44 & 27 & 55.10 & \\
\hline \multicolumn{7}{|l|}{ TNM stage } \\
\hline $\mathrm{I}+\mathrm{II}$ & 52 & 7 & 77.78 & 45 & 91.84 & 0.203 \\
\hline $\mathrm{III}+\mathrm{IV}$ & 6 & 2 & 22.22 & 4 & 8.16 & \\
\hline \multicolumn{7}{|l|}{ Milan criteria (21) } \\
\hline Within criteria & 24 & 2 & 22.22 & 22 & 44.90 & 0.204 \\
\hline Beyond criteria & 34 & 7 & 77.78 & 27 & 55.10 & \\
\hline \multicolumn{7}{|l|}{ UCSF criteria (22) } \\
\hline Within criteria & 31 & 3 & 33.33 & 28 & 57.14 & 0.188 \\
\hline Beyond criteria & 27 & 6 & 66.67 & 21 & 42.86 & \\
\hline \multicolumn{7}{|c|}{ Hangzhou criteria (23) } \\
\hline Within criteria & 46 & 6 & 66.67 & 40 & 81.63 & 0.308 \\
\hline Beyond criteria & 12 & 3 & 33.33 & 9 & 18.37 & \\
\hline
\end{tabular}

${ }^{\mathrm{a}} \mathrm{P}<0.05$ was considered to indicate statistical significance a statistically significant difference, according to the $\chi^{2}$ test. HCC, hepatocellular carcinoma; HBV, hepatitis B virus; PVTT, portal vein tumor thrombosis; AFP, $\alpha$-fetoprotein; TNM, tumor-node-metastasis; UCSF, University of California, San Francisco.

higher recurrence rate $(\mathrm{P}=0.004)$ (Table III). Thus, these data indicate that high PVT1 expression levels in HCC may be associated with disease progression.

PVT1 predicts HCC recurrence following liver transplantation. To determine whether PVT1 could be employed as a prognostic biomarker for HCC, clinical data of HCC cohort two were analyzed in detail. Using the cut-off value, the 214 patients were divided into two groups: A low-expression group ( $\mathrm{n}=57)$ and a high-expression group $(\mathrm{n}=157)$. Kaplan-Meier analysis indicated that the patients with high PVT1 expression levels had a poor RFS period ( $\mathrm{P}=0.021$; Fig. $4 \mathrm{~A})$. Although no statistical 
Table III. Clinicopathological correlation of PVT1 expression in human HCC (cohort two).

\begin{tabular}{|c|c|c|c|c|c|c|}
\hline \multirow[b]{2}{*}{ Factor } & \multirow[b]{2}{*}{ Cases, $\mathrm{n}$} & \multicolumn{2}{|c|}{ Low PVT1 expression } & \multicolumn{2}{|c|}{ High PVT1 expression } & \multirow[b]{2}{*}{ P-value } \\
\hline & & $\mathrm{n}$ & $\%$ & $\mathrm{n}$ & $\%$ & \\
\hline \multicolumn{7}{|l|}{ Age, years } \\
\hline$\leq 60$ & 187 & 49 & 85.96 & 138 & 87.90 & 0.707 \\
\hline$>60$ & 27 & 8 & 14.04 & 19 & 12.10 & \\
\hline \multicolumn{7}{|l|}{ Gender } \\
\hline Female & 19 & 4 & 7.02 & 15 & 9.55 & 0.564 \\
\hline Male & 195 & 53 & 92.98 & 142 & 90.45 & \\
\hline \multicolumn{7}{|l|}{$\mathrm{HBV}$} \\
\hline Negative & 5 & 2 & 3.51 & 3 & 1.91 & 0.494 \\
\hline Positive & 210 & 55 & 96.49 & 154 & 98.09 & \\
\hline \multicolumn{7}{|l|}{ Cirrhosis } \\
\hline No & 2 & 1 & 1.75 & 1 & 0.64 & 0.453 \\
\hline Yes & 212 & 56 & 98.25 & 156 & 99.36 & \\
\hline \multicolumn{7}{|l|}{ Tumor size, $\mathrm{cm}$} \\
\hline$\leq 5$ & 122 & 33 & 57.89 & 89 & 56.69 & 0.875 \\
\hline$>5$ & 92 & 24 & 42.11 & 68 & 43.31 & \\
\hline \multicolumn{7}{|l|}{ Tumor number } \\
\hline Single & 89 & 28 & 49.12 & 61 & 38.85 & 0.178 \\
\hline Multiple & 125 & 29 & 50.88 & 96 & 61.15 & \\
\hline \multicolumn{7}{|l|}{ PVTT } \\
\hline Absent & 142 & 40 & 70.18 & 102 & 64.97 & 0.476 \\
\hline Present & 72 & 17 & 29.82 & 55 & 35.03 & \\
\hline \multicolumn{7}{|c|}{ Preoperative AFP level, $\mathrm{ng} / \mathrm{ml}$} \\
\hline$\leq 400$ & 108 & 37 & 64.91 & 71 & 45.22 & $0.011^{\mathrm{a}}$ \\
\hline$>400$ & 106 & 20 & 35.09 & 86 & 54.78 & \\
\hline \multicolumn{7}{|c|}{ Histopathological grading } \\
\hline Well + moderately & 110 & 35 & 61.40 & 75 & 47.77 & 0.078 \\
\hline Poorly & 104 & 22 & 38.60 & 82 & 52.23 & \\
\hline \multicolumn{7}{|l|}{ TNM stage } \\
\hline $\mathrm{I}+\mathrm{II}$ & 99 & 30 & 52.63 & 69 & 43.95 & 0.260 \\
\hline III + IV & 115 & 27 & 47.37 & 88 & 56.05 & \\
\hline \multicolumn{7}{|l|}{ Recurrence } \\
\hline No & 100 & 36 & 63.16 & 64 & 40.76 & $0.004^{\mathrm{a}}$ \\
\hline Yes & 114 & 21 & 36.84 & 93 & 59.24 & \\
\hline \multicolumn{7}{|l|}{ Milan criteria (19) } \\
\hline Within criteria & 75 & 24 & 42.11 & 51 & 32.48 & 0.192 \\
\hline Beyond criteria & 139 & 33 & 57.89 & 106 & 67.52 & \\
\hline \multicolumn{7}{|l|}{ UCSF criteria (20) } \\
\hline Within criteria & 93 & 27 & 47.37 & 66 & 42.04 & 0.487 \\
\hline Beyond criteria & 121 & 30 & 52.63 & 91 & 57.96 & \\
\hline \multicolumn{7}{|c|}{ Hangzhou criteria (21) } \\
\hline Within criteria & 105 & 31 & 54.39 & 74 & 47.13 & 0.348 \\
\hline Beyond criteria & 109 & 26 & 45.61 & 83 & 52.87 & \\
\hline
\end{tabular}

${ }^{a} \mathrm{P}<0.05$ was considered to indicate a statistically significant difference, according to the $\chi^{2}$ test. HCC, hepatocellular carcinoma; HBV, hepatitis B virus; PVTT, portal vein tumor thrombosis; AFP, serum $\alpha$-fetoprotein; TNM, tumor-node-metastasis; UCSF, University of California, San Francisco.

significance of PVT1 expression as a predictor of overall survival was determined, patients exhibiting high PVT1 expression levels demonstrated a trend for poor prognoses (Fig. 4B; $\mathrm{P}=0.464$ ).
To identify the risk factors associated with post-transplant RFS, 11 clinicopathological factors were evaluated by performing Cox univariate and multivariate analyses. 

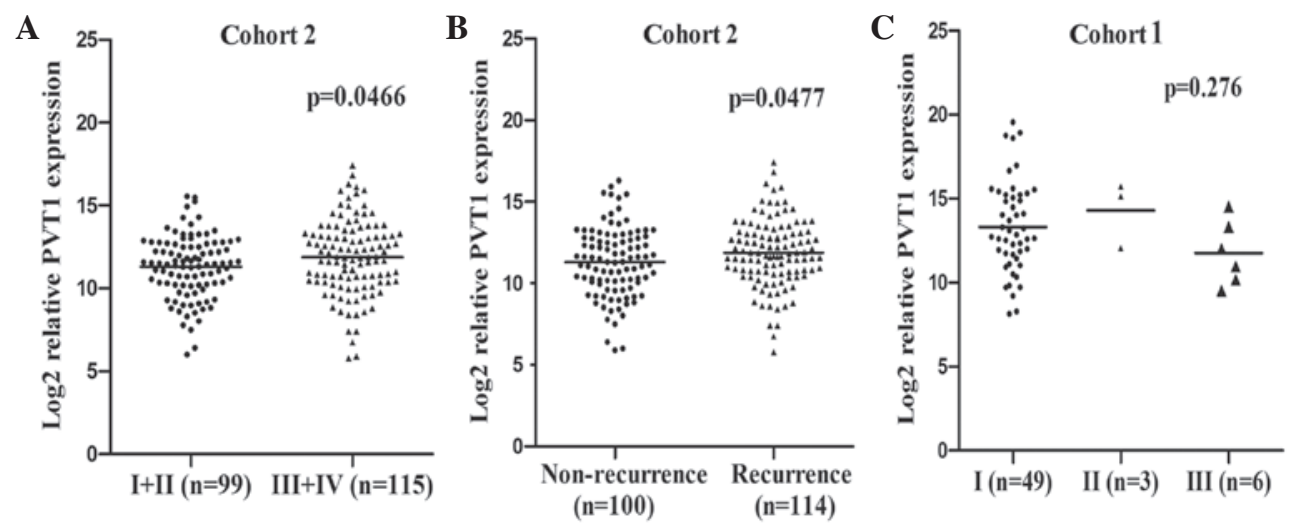

Figure 3. PVT1 expression levels defined according to the tumor-node-metastasis stage and recurrence status. (A) Cohort two: Early (stages I + II) vs. advanced stage (stages III + IV). (B) Cohort two: Non-recurrence vs. recurrence. (C) Cohort one: Three different stages (I vs. II vs. III). PVT1 expression levels were normalized to an internal control and $\log 2$-transformed. Horizontal lines in the plots represent the mean values. P-values were analyzed using (A and B) the independent $\mathrm{t}$-test and $(\mathrm{C})$ one-way analysis of variance.
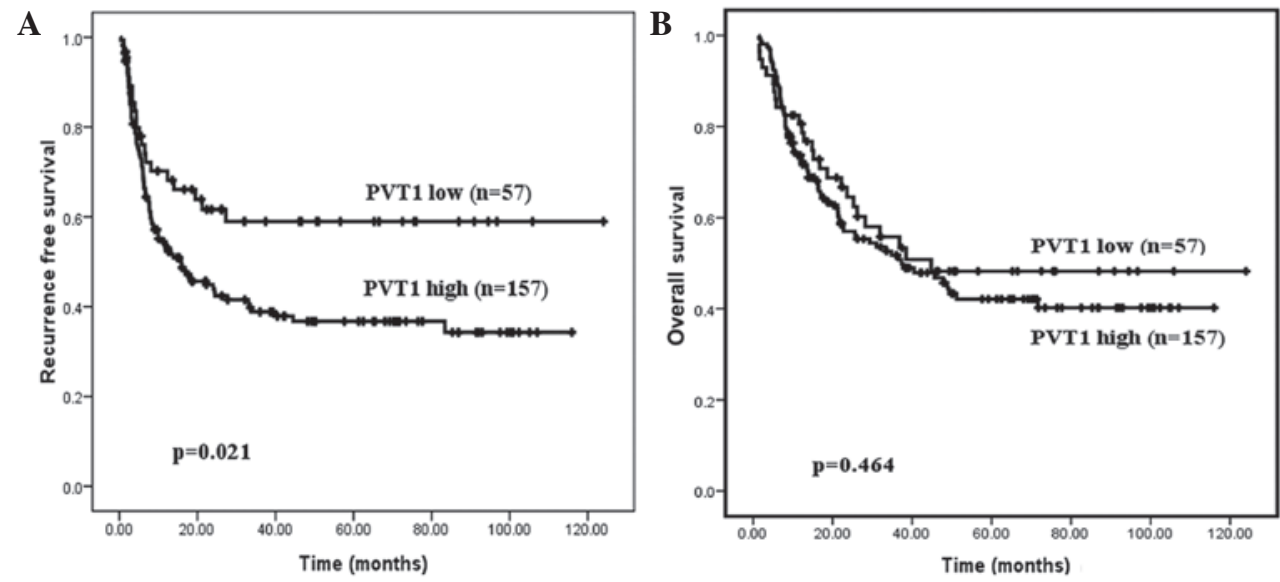

Figure 4. Kaplan-Meier survival curves according to PVT1 level. Patients with high PVT1 expression had poor (A) recurrence free survival and (B) overall survival rates. P-values were calculated using the log-rank test.

Univariate analysis demonstrated that the significant prognostic factors for HCC recurrence were tumor size, tumor number, histopathological grade, PVTT, preoperative AFP level, TNM stage and PVT1 expression (all $\mathrm{P}<0.05$ ). Only tumor size (HR, 2.462; 95\% CI, 1.652-3.671; $\mathrm{P}<0.001)$, tumor number (HR, 1.802; 95\% CI, 1.194-2.719; $\mathrm{P}=0.005)$, PVTT (HR, 2.075, 95\% CI, 1.418-3.037; P<0.001), preoperative AFP level (HR, 1.539;95\% CI, 1.027-2.305; $\mathrm{P}=0.037)$ and PVT1 expression (HR, 1.653; 95\% CI, 1.019-2.681; $\mathrm{P}=0.042$ ) were identified as independent prognostic factors associated with tumor recurrence following liver transplantation, as determined by the Cox multivariate analysis (Table IV).

\section{Discussion}

The complexity of the human transcriptome has been highlighted by various high-throughput studies $(24,25) ; \leq 70 \%$ of the genome is transcribed into RNA that does not act as templates for protein (26). The non-protein-coding portion of the genome constitutes the vast majority of genomic information, as well as exhibiting critical functional roles (27). Improved understanding of the aberrant expression patterns, cellular functions and underlying mechanisms of the non-protein-coding genome may aid in expanding the current understanding of the complex regulatory network in cancer biology.

Although the majority of previous studies have focused on short RNAs in cancer research, such as miRNAs, IncRNAs are gaining prominence. A number of classic lncRNAs have been implicated in human hepatocarcinogenesis, exhibiting oncogenic or tumor suppressive roles. One such example of oncogenic lncRNA is HOTAIR, which was initially identified in foreskin fibroblasts. HOTAIR resides in the HOX C locus, acting as a modular scaffold to recruit the polycomb repressive complex 2 to specific target sequences that ultimately results in the suppression of numerous genes (28). Our previous study demonstrated that the expression of HOTAIR is upregulated in HCC tissues compared with paired non-cancerous tissues, and high expression levels of HOTAIR were an independent prognostic marker for HCC recurrence and shorter survival (10). An additional classic oncogenic lncRNA is MALAT1, which regulated the alternative splicing of a subset of pre-mRNAs to promote cancer metastasis (29). In our previous study, MALAT1 was unregulated in HCC and served as a negative prognostic factor for tumor progression and patient survival (12). 
Table IV. Cox univariate and multivariate analysis of predictors of recurrence in hepatocellular carcinoma patients following liver transplant.

\begin{tabular}{|c|c|c|c|c|c|c|}
\hline \multirow[b]{2}{*}{ Variable for tumor recurrence } & \multicolumn{3}{|c|}{ Univariate analysis } & \multicolumn{3}{|c|}{ Multivariate analysis } \\
\hline & HR & $95 \% \mathrm{CI}$ & P-value & HR & $95 \% \mathrm{CI}$ & P-value \\
\hline Age, years (>60 vs. $\leq 60)$ & 0.669 & $0.349-1.280$ & 0.224 & & & \\
\hline Gender (male vs. female) & 1.577 & $0.768-3.239$ & 0.214 & & & \\
\hline $\mathrm{HBV}$ & 21.091 & $0.159-2793$ & 0.221 & & & \\
\hline Cirrhosis & 1.039 & $0.145-7.443$ & 0.970 & & & \\
\hline Tumor size, $\mathrm{cm}(>5$ vs. $\leq 5)$ & 3.431 & $2.340-5.029$ & 0.000 & 2.462 & $1.652-3.671$ & $0.000^{\mathrm{a}}$ \\
\hline Tumor number (multiple vs. single) & 2.393 & $1.597-3.582$ & 0.000 & 1.802 & $1.194-2.719$ & $0.005^{\mathrm{a}}$ \\
\hline Histopathological grade (poorly vs. well + moderately) & 1.665 & $1.148-2.415$ & 0.007 & & & \\
\hline PVTT (present vs. absent) & 2.826 & $1.947-4.102$ & 0.000 & 2.075 & $1.418-3.037$ & $0.000^{\mathrm{a}}$ \\
\hline Preoperative AFP level, ng/ml (>400 vs. $\leq 400)$ & 2.380 & $1.622-3.492$ & 0.000 & 1.539 & $1.027-2.305$ & $0.037^{\mathrm{a}}$ \\
\hline TNM stage (III + IV vs. I + II) & 4.584 & $2.987-7.034$ & 0.000 & & & \\
\hline PVT1 expression (high vs. low) & 1.738 & $1.082-2.792$ & 0.022 & 1.653 & $1.019-2.681$ & $0.042^{\mathrm{a}}$ \\
\hline
\end{tabular}

${ }^{\text {a }} \mathrm{P}<0.05 \mathrm{HR}$, hazard ratio; CI, confidence interval; HBV, hepatitis B virus; PVTT, portal vein tumor thrombosis; AFP, $\alpha$-fetoprotein; TNM, tumor-nodemetastasis.

Furthermore, tumor suppressive lncRNAs may affect various tumor suppressor pathways. For example, MEG3 was identified to be frequently downregulated in HCC by miR29a-mediated promoter methylation, and MEG3 inhibited cell growth by functionally interacting with the p53 signaling pathway (13). Additionally, H19 associated with the protein complex heterogeneous nuclear ribonucleoprotein U/P300/cAMP-response element binding protein-associated factor/RNA polymerase II, epigenetically activating the miR-200 family, and thus suppressing HCC metastasis (14).

Various reports have presented evidence that PVT1 contributes to cancer pathophysiology. For example, PVT1 was markedly overexpressed in colorectal (15), ovarian and breast cancers (16). In the current study, PVT1 expression was initially analyzed in 58 pairs of HCC resection samples (cohort one), and it was identified that PVT1 was significantly upregulated in HCC. Subsequently, the PVT1 expression level from an independent cohort of 214 HCC patients (cohort two) was analyzed. The upregulation of PVT1 was validated in this independent cohort. The findings of the present study indicate that PVT1 is predominantly overexpressed in HCC tissues, regardless of the type of surgical intervention that the patients undergo. However, the molecular mechanism underlying the upregulation of PVT1 remains poorly understood. Guan et al (16) demonstrated that amplification of chromosome 8q24 increased the expression of PVT1 in ovarian and breast cancers. Consistent with this finding, Takahashi et al (15) identified that chromosome 8q24 copy number gain promoted the expression of PVT1 in colorectal cancer. Additional studies are required to determine if PVT1 is similarly regulated in HCC.

Furthermore, the present study identified that PVT1 was more likely to be overexpressed in advanced-stage and recurrence patients. Correlation analysis indicated that increased expression of PVT1 was associated with a higher AFP level and a higher recurrence rate. These data support the hypothesis that PVT1 is associated with disease progression. In addition, survival analysis demonstrated that the patients with high PVT1 expression exhibited poor RFS. Multivariate analysis identified that PVT1 was an independent prognostic factor for RFS. Therefore, data from the present study indicate that PVT1 may be a novel biomarker for risk surveillance and adjuvant therapy screening of HCC patients following liver transplantation. Furthermore, overexpression of PVT1 may be used by surgeons to identify high-risk patients who may benefit from preventive strategies as opposed to surgery.

The effects and precise molecular mechanisms underlying the altered expression of PVT1 in HCC are unclear. Guan et al (16) demonstrated that depletion of PVT1 may decrease cell proliferation and increase cell apoptosis in ovarian and breast cancer cell lines. Similarly, Takahashi et al (15) identified that PVT1 knockdown promotes apoptosis in colorectal cancer cell lines via the TGF- $\beta$ signaling pathway. Thus, the detailed mechanism is likely to be complex. Barsotti et al (30) identified PVT1 as a p53-inducible target gene. Furthermore, the PVT1 locus produced numerous spliced non-coding RNAs, as well as six miRNAs, which may be a molecular switch for cell life and death. Further experiments are required to elucidate the detailed interplay.

In conclusion, the present study demonstrated that PVT1 was overexpressed in two independent human HCC cohorts and 10 liver cancer cell lines. Increased expression levels of PVT1 were associated with a higher AFP level and a higher recurrence rate. Furthermore, PVT1 served as an independent prognostic factor for RFS. Thus, the findings of the present study indicate that PVT1 may act as a novel biomarker for predicting tumor recurrence in HCC patients and may be a potential therapeutic target.

\section{Acknowledgements}

This abstract was presented at the American College of Surgeons Clinical Congress (October 26-October 30, 2014), San Francisco, CA, USA, and was published as abstract S23 in the Journal of the American College of Surgeons 219: S3, 2014. The present study was supported by grants from the National 
High Technology Research and Development Program of China (863 Program), the Special Fund for Health Research in the Public Welfare, the Zhejiang Provincial Natural Science Foundation for Young Distinguished Scholars, the National Science and Technology Major Project and the Foundation for Innovative Research Groups of the National Natural Science Foundation of China (grant nos. 2012AA021002, 201302009, R2110125, 2012ZX10002017 and 81121002, respectively). The authors of the present study would also like to thank all of the patients enrolled in the study for their support.

\section{References}

1. Jemal A, Bray F, Center MM, et al: Global cancer statistics. CA Cancer J Clin 61: 69-90, 2011.

2. El-Serag HB and Rudolph KL: Hepatocellular carcinoma: epidemiology and molecular carcinogenesis. Gastroenterology 132: 2557-2576, 2007.

3. Cha C, Fong Y, Jarnagin WR, Blumgart LH and DeMatteo RP: Predictors and patterns of recurrence after resection of hepatocellular carcinoma. J Am Coll Surg 197: 753-758, 2003.

4. Shah SA, Cleary SP, Wei AC, et al: Recurrence after liver resection for hepatocellular carcinoma: risk factors, treatment, and outcomes. Surgery 141: 330-339, 2007.

5. Zimmerman MA, Ghobrial RM, Tong MJ, et al: Recurrence of hepatocellular carcinoma following liver transplantation: a review of preoperative and postoperative prognostic indicators. Arch Surg 143: 182-188; discussion 188, 2008.

6. Wilusz JE, Sunwoo H and Spector DL: Long noncoding RNAs: functional surprises from the RNA world. Genes Dev 23 1494-1504, 2009.

7. Mercer TR, Dinger ME and Mattick JS: Long non-coding RNAs: insights into functions. Nat Rev Genet 10: 155-159, 2009.

8. Tsai MC, Spitale RC and Chang HY: Long intergenic noncoding RNAs: new links in cancer progression. Cancer Res 71: 3-7, 2011

9. Wang KC and Chang HY: Molecular mechanisms of long noncoding RNAs. Mol Cell 43: 904-914, 2011.

10. Yang Z, Zhou L, Wu LM, et al: Overexpression of long non-coding RNA HOTAIR predicts tumor recurrence in hepatocellular carcinoma patients following liver transplantation. Ann Surg Oncol 18: 1243-1250, 2011.

11. Ishibashi M, Kogo R, Shibata K, et al: Clinical significance of the expression of long non-coding RNA HOTAIR in primary hepatocellular carcinoma. Oncol Rep 29: 946-950, 2013.

12. Lai MC, Yang Z, Zhou L, et al: Long non-coding RNA MALAT-1 overexpression predicts tumor recurrence of hepatocellular carcinoma after liver transplantation. Med Oncol 29: 1810-1816, 2012.

13. Braconi C, Kogure T, Valeri N, et al: microRNA-29 can regulate expression of the long non-coding RNA gene MEG3 in hepatocellular cancer. Oncogene 30: 4750-4756, 2011.
14. Zhang L, Yang F, Yuan JH, et al: Epigenetic activation of the MiR-200 family contributes to H19-mediated metastasis suppression in hepatocellular carcinoma. Carcinogenesis 34: 577-586, 2013.

15. Takahashi Y, Sawada G, Kurashige J, et al: Amplification of PVT-1 is involved in poor prognosis via apoptosis inhibition in colorectal cancers. Br J Cancer 110: 164-171, 2014.

16. Guan Y, Kuo WL, Stilwell JL, et al: Amplification of PVT1 contributes to the pathophysiology of ovarian and breast cancer. Clin Cancer Res 13: 5745-5755, 2007.

17. Schlaeger C, Longerich T, Schiller C, et al: Etiology-dependent molecular mechanisms in human hepatocarcinogenesis. Hepatology 47: 511-520, 2008.

18. Ding J, Huang S, Wu S, et al: Gain of miR-151 on chromosome 8q24.3 facilitates tumour cell migration and spreading through downregulating RhoGDIA. Nat Cell Biol 12: 390-399, 2010.

19. Edmondson HA and Steiner PE: Primary carcinoma of the liver: a study of 100 cases among 48,900 necropsies. Cancer 7: 462-503, 1954

20. Greene FL, Page DL, Fleming ID, et al (eds): Liver. In: AJCC Cancer Staging Manual. 6th edition. Springer, Chicago, IL, p435, 2002.

21. Mazzaferro V, Regalia E, Doci R, et al: Liver transplantation for the treatment of small hepatocellular carcinomas in patients with cirrhosis. N Engl J Med 334: 693-699, 1996.

22. Yao FY, Ferrell L, Bass NM, et al: Liver transplantation for hepatocellular carcinoma: expansion of the tumor size limits does not adversely impact survival. Hepatology 33: 1394-1403, 2001.

23. Zheng SS, Xu X, Wu J, et al: Liver transplantation for hepatocellular carcinoma: Hangzhou experiences. Transplantation 85: 1726-1732, 2008.

24. Gibb EA, Vucic EA, Enfield KS, et al: Human cancer long non-coding RNA transcriptomes. PLoS One 6: e25915, 2011.

25. Prensner JR, Iyer MK, Balbin OA, et al: Transcriptome sequencing across a prostate cancer cohort identifies PCAT-1, an unannotated lincRNA implicated in disease progression. Nat Biotechnol 29: 742-749, 2011

26. Gutschner T and Diederichs S: The hallmarks of cancer: a long non-coding RNA point of view. RNA Biol 9: 703-719, 2012.

27. Guttman M and Rinn JL: Modular regulatory principles of large non-coding RNAs. Nature 482: 339-346, 2012.

28. Rinn JL, Kertesz M, Wang JK, et al: Functional demarcation of active and silent chromatin domains in human HOX loci by noncoding RNAs. Cell 129: 1311-1323, 2007.

29. Tripathi V, Ellis JD, Shen Z, et al: The nuclear-retained noncoding RNA MALAT1 regulates alternative splicing by modulating SR splicing factor phosphorylation. Mol Cell 39: 925-938, 2010

30. Barsotti AM, Beckerman R, Laptenko O, et al: p53-dependent induction of PVT1 and miR-1204. J Biol Chem 287: 2509-2519, 2012. 